\title{
Spinocerebellar ataxia type 14: study of a family with an exon 5 mutation in the PRKCG gene
}

\author{
M C Fahey*, M A Knight*, J H Shaw, R J McK Gardner, D du Sart, P J Lockhart, M B Delatycki, \\ P C Gates, É Storey
}

We report our observations in an Australian family with spinocerebellar ataxia type 14 (SCA 14). We describe a novel mutation in exon 5 of the PRKCG gene, altering a highly conserved cysteine to a phenylalanine at codon 150 , and record the detailed clinical observations in six affected family members.

$\mathrm{T}$ he autosomal dominant cerebellar ataxias (ADCA) are clinically categorised according to the presence or absence of extracerebellar features, and genetically classified according to their chromosomal locus. In some the gene has been identified, while in others chromosomal mapping has defined a region within which the gene is yet to be found. These are designated spinocerebellar ataxias (SCAs) and are assigned a number corresponding to their order of discovery, the most recent being SCA25. ${ }^{1}$ In 11 of these the gene has been identified, 10 of which involve a trinucleotide or pentanucleotide repeat, and one of which, SCA 14, is due to classical types of mutation in the protein kinase C gamma (PRKCG) gene, mostly in exon $4 .{ }^{23}$ We have studied an Australian family with SCA 14 due to a novel mutation in exon 5 of the PRKCG gene.

\section{METHODS \\ Patients}

Fifteen living family members were interviewed and examined by ES, MCF, and RJMCKG in a standardised fashion, and three family members had brain MRI scanning. Consent was obtained from family members and their spouses and blood samples were drawn. Genomic DNA was extracted from blood leucocytes using the Amersham Nucleon BACC2 for Blood and Cell Cultures Kit (Amersham International, Buckinghamshire, UK). After demonstration of linkage to the SCA 14 locus, exons 4 and 5 of the PRKCG gene were amplified from genomic DNA and sequenced using published primers. Sequencing was performed directly on this PCR product for two affected family members. Following mutation detection, all family members were invited to attend genetic counselling. The study was approved by the Women's and Children's Hospital Human Ethics Committee (Melbourne).

\section{RESULTS}

\section{Clinical features}

Six affected family members (five females, one male) were identified from two generations. The average age of onset was 31.3 years (range 19-44) and average disease duration when seen was 14.3 years (range 4-31). Symptoms and signs are summarised in table 1 . The first symptom was gait imbalance in five, and combined gait imbalance and dysarthria in one. At the time of review all were ambulant without walking aids and living independently. Progress of the condition had been slow; some had been symptomatic for decades.

Examination revealed cerebellar ataxia with abnormal eye movements and brisk reflexes. Mild or moderate cerebellar ataxia was present in the upper and lower limbs, with all affected patients showing signs of dysmetria, overshoot on ballistic tracking movements, abnormal ramp tracking in the upper limbs, and dyssynergia and dysmetria in the lower limbs. Finger tapping was irregular, but in only one individual was it more than 1 SD below age, sex, and education adjusted normal rates. One patient displayed a slight truncal tremor on standing, but no other adventitious movements were evident.

Eye movements were abnormal in all six affected individuals. The most striking feature was hypermetric saccades into downgaze. Five of the six subjects also exhibited hypermetric horizontal saccades. Two of the six demonstrated downbeat nystagmus in the primary position on ophthalmoscopy, and one of these and one other displayed upwards gaze evoked nystagmus on upgaze. Smooth pursuit eye movements were mildly broken up in four. Visual suppression of the vestibulo-ocular reflex (VOR) was impaired in five, especially vertically. The VOR gain, as judged by dynamic $v$ static visual acuity and ophthalmoscopy during head oscillation, was uniformly normal. Colour vision, including in the tritan axis, was normal.

All had at least mild hyperreflexia, with evidence of abnormal reflex spread in the upper and/or lower limbs in four of the six subjects. Two had a mild spastic catch at the knees (Modified Ashworth Scale grade 1), but ankle clonus was absent and Babinski reflexes were downgoing in all. Two of the six subjects demonstrated decreased distal pinprick sensation in the lower limbs. Two point discrimination was also mildly impaired $(\geqslant 2 \mathrm{~cm})$ in the great toes in two. However, lower limb motor and sensory nerve conduction studies and F waves were entirely normal in two (cases 4 and 5 ), including one who had demonstrated mildly impaired two point discrimination. MRI images from a representative affected person are shown in fig 1 .

\section{Genotyping, linkage, and mutation analysis}

Direct repeat expansion testing excluded SCAs 1-3, 6-8, and 17 in representative family members. The two point linkage analysis results for the mapped candidate loci for SCAs 4-6, $10-12,15-16$, and 18-22 revealed no evidence of linkage (data not shown). Minimal linkage analysis indicated linkage to the chromosomal region 19q13.3-q13.4, which includes the loci of SCA 13 and SCA 14 (data not shown). We

Abbreviations: $A D C A$, autosomal dominant cerebellar ataxia; PRKCG gene, protein kinase $C$ gamma gene; SCA, spinocerebellar ataxia; VOR, vestibulo-ocular reflex 
Table 1 Clinical observations in six members of the Australian SCA 14 family

\begin{tabular}{|c|c|c|c|c|c|c|c|}
\hline Case & 1 & 2 & 3 & 4 & 5 & 6 & (Average) \\
\hline Sex & $\mathrm{F}$ & $\mathrm{F}$ & $\mathrm{F}$ & $\mathrm{F}$ & $\mathrm{F}$ & $M$ & \\
\hline Age at examination & 53 & 50 & 45 & 50 & 45 & 31 & $(45.6)$ \\
\hline Age of symptom onset & 40 & 44 & 28 & 19 & 30 & 27 & (31.3) \\
\hline Duration of symptoms (years) & 13 & 6 & 17 & 31 & 15 & 4 & (14.3) \\
\hline Gait ataxia* & ++ & + & + & ++ & ++ & + & \\
\hline Appendicular ataxia** & + & + & + & + & + & + & \\
\hline Dysarthria§ & ++ & + & \pm & \pm & ++ & ++ & \\
\hline Hyperreflexia§§ & + & + & + & ++ & + & ++ & \\
\hline Nystagmus & $+^{*}$ & - & - & $+\dagger$ & $+\ddagger$ & - & \\
\hline Broken-up smooth pursuit & $\downarrow \uparrow \leftrightarrow$ & $\mathrm{N}$ & $\downarrow$ & $\downarrow \uparrow \leftrightarrow$ & $\leftrightarrow$ & $\mathrm{N}$ & \\
\hline Impaired visual suppression VOR & $\downarrow \uparrow \leftrightarrow$ & $\downarrow \uparrow$ & $\downarrow \uparrow$ & $\downarrow \uparrow \leftrightarrow$ & $\downarrow \uparrow \leftrightarrow$ & $\mathrm{N}$ & \\
\hline Saccadic hypermetria & $\downarrow \leftrightarrow$ & $\downarrow$ & $\downarrow \leftrightarrow$ & $\downarrow \leftrightarrow$ & $\downarrow \leftrightarrow$ & $\downarrow \leftrightarrow$ & \\
\hline
\end{tabular}

*Gait ataxia: \pm : evident only on hopping within a $30 \mathrm{~cm}$ circle; +: difficulty turning and/or tandem gait only; ++: ataxia evident on ordinary walking, no aids required; +++: gait aids required.

**Appendicular ataxia: +: mild; ++: moderate; +++: marked.

§Dysarthria: \pm : evident on rapid "la la la ..." only; + : difficulty on test phrases only; ++: evident on routine conversation but full intelligibility preserved; +++: intelligibility impaired.

$\S \S$ Hyperreflexia: +: mild; ++: moderate, with reflex spread from biceps to finger flexors and/or crossed adductor responses; +++: marked, with clonus and/or extensor plantar responses.

-Nystagmus: +: present; *: upbeat in upgaze; †: upbeat in upgaze, downbeat in primary position (ophthalmoscopic observation); $\ddagger$ : downbeat in primary position (ophthalmoscopic observation); -: not present.

Eye movements: arrows indicate that the particular abnormal response occurred in downgaze, upgaze, or lateral gaze.

$\mathrm{N}$, no abnormal response; VOR, vestibulo-ocular reflex.

proceeded to analysis of the SCA 14 gene, $P R K C G$, since the clinical phenotype in our family was consistent with this diagnosis.

Two substitutions in highly conserved, adjacent nucleotides were identified within exon 5 in the affected family members: GC to TT at positions 635 and 636 of the mRNA (GenBank Accession NM_002739). These comprise the second and third nucleotides of codon 150, TGC, normally coding for cysteine, and this mutation to TTT is predicted to lead to substitution with phenylalanine (C150F). This two nucleotide substitution co-segregated faithfully with the disease phenotype. Analysis of DNA from two sets of parents of two affected individuals demonstrated segregation of the double mutation with affected individuals. The mutation was not identified in 290 control chromosomes.

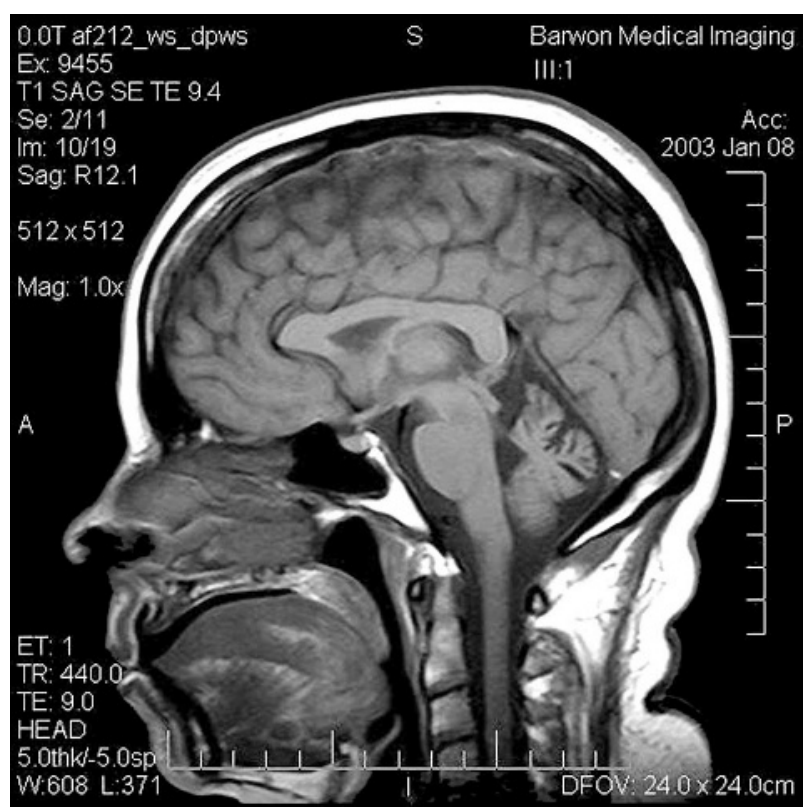

Figure $1 \mathrm{MRI}$ image (sagittal T1) from case 4. The cerebellar atrophy is most prominent in the superior and mid-vermis, with only slight involvement of the hemispheres. The MRI findings were similar in two other patients imaged, although megacisterna magna, a presumed unrelated congenital abnormality, was demonstrated in one.

\section{DISCUSSION}

This is the tenth reported family with SCA 14. There is little detailed information published on the SCA 14 phenotype, and we have therefore recorded our clinical observations in full (table 1). The clinical picture is of a slowly progressive, pure cerebellar ataxia, with hyperreflexia without spasticity or extensor plantar response, and abnormalities of eye movement.

In the 10 families on record, a pure cerebellar ataxia is described in most. ${ }^{2-7}$ In one family dysphagia was noted, and diplopia was present in one sporadic case. ${ }^{3}$ Hyperreflexia was observed in two families, ${ }^{3}{ }^{4}$ as well as in ours. Extracerebellar signs have been described, particularly in those with a younger age of onset, and it is possible that these features reflect particular genotype-phenotype correlations. In the first family reported, Yamashita et $a l^{5}$ described axial myoclonus in some younger affected persons, while an extrapyramidal component (focal dystonia) was observed in two having an onset in their twenties in the family of van de Warrenburg et al. ${ }^{7}$

While eye movement abnormalities are a feature of many SCAs, when combined with other clinical features, they may help distinguish SCA 14 from other slowly progressive pure cerebellar ataxias. In our family, hypermetria of saccades in downgaze was present from the early stages of the disease, while nystagmus was a more prominent feature in those with longer duration of illness. In other series, Yamashita et $a l^{5}$ described ocular overshoot (saccadic hypermetria?) in three out of nine patients, while Brkanac et al ${ }^{4}$ recorded horizontal jerk (gaze evoked) nystagmus or broken-up smooth pursuit in the majority, although it is not clear whether saccadic hypermetria was specifically sought.

Hypermetric saccades may also be seen in SCA $1,{ }^{8}{ }^{9}$ SCA $2,{ }^{9}$ SCA $6,{ }^{10}$ and SCA $20,{ }^{11}$ but other features may distinguish these diseases from SCA 14. SCA 1 is often associated with supranuclear gaze palsy, spasticity, sensory loss, and dysphagia as a reflection of brain stem atrophy. ${ }^{9}$ SCA 2 is more usually associated with slow saccades ${ }^{9}$ and hyporeflexia. SCA 6 may not be distinguishable clinically, but can be diagnosed with a readily available genetic test. SCA 20 is associated with dentate calcification and palatal tremor. ${ }^{11}$ SCA 8 is also associated with saccadic dysmetria in moderately affected individuals, although the direction and type are not defined. ${ }^{12}$ SCA $11^{13}$ is associated with horizontal and vertical nystagmus 
and jerky pursuit. SCA $16^{14}$ is associated with horizontal gaze evoked nystagmus, but other specific details of eye movement abnormalities are not mentioned. SCA 15, another slowly progressive pure cerebellar ataxia, is not associated with abnormal eye movements. ${ }^{15}$

The dorsal cerebellar vermis (lobules V, VI, and VII) and the fastigial nuclei are important for saccade control. Chemical inhibition of the fastigial nuclei leads to hypermetric saccades through influence on the saccade burst generator within the brainstem. ${ }^{16}$ This would be consistent with the MRI findings in our family.

Until recently, repeat expansions were the only mutational mechanism implicated in the SCA phenotypes, but SCA 14 is one of two new exceptions to this rule. Van Swieten et al described a missense mutation in the FGF14 gene in a family with an ADCA that has not been assigned an SCA number. ${ }^{17}$ SCA 14 is due to mutation (missense, small deletion, splice site) in the PRKCG gene..$^{367}$ Most mutations have been in the cysteine rich region of the gene, and most of these fall within the region coded by exon 4 , in the Cys 2 domain. This family is the first with an exon 5 mutation, the altered codon, number 150, being the last one of the Cys2 domain. This codon is highly conserved, and normally codes for cysteine; the TGC $\rightarrow$ TTT mutation introduces phenylalanine. PRKCG strongly affects and regulates cerebellar Purkinje cell dendritic development, suggesting an important role for protein kinase $\mathrm{C}$ in activity dependent Purkinje cell maturation. ${ }^{18}$ In vivo studies show that the $\mathrm{Cl} 150 \mathrm{~F}$ change is functionally significant as it increases PKC gamma kinase activity when compared to wild type PKC gamma in COS-7 cells. ${ }^{19}$

While Huntington disease has been the model for predictive gene testing in the adult onset neurodegenerative disorders, counselling with respect to SCA 14 can be adjusted to take into account the considerably milder picture in terms of functional neurological disability, and the apparent absence of any effect upon cognitive ability. As further knowledge is gained on genotype-phenotype correlation, it may be appropriate to offer tailored advice according to the precise mutation.

\section{ACKNOWLEDGEMENTS}

The authors are grateful to the family members for their willing cooperation.

\section{Authors' affiliations}

J H Shaw, R J McK Gardner, D du Sart, E Storey, Genetic Health Services Victoria, Melbourne, Australia

M C Fahey, M A Knight, Murdoch Childrens Research Institute, Royal Children's Hospital, Melbourne, Australia

P J Lockhart, M B Delatycki, Bruce Lefroy Centre for Genetic Health Research, Royal Children's Hospital, Melbourne, Australia

P C Gates, Department of Neuroscience, Barwon Health, Geelong, Australia

E Storey, Department of Medicine (Neuroscience), Alfred Hospital Campus of Monash University, Melbourne, Australia

The study was supported by the Murdoch Childrens Research Institute.
Competing interests: none declared

*These authors contributed equally to this work.

The subjects mentioned in this paper have agreed to their details being published.

Correspondence to: Professor Elsdon Storey, Department of Neuroscience, Monash University (Alfred Hospital Campus), Commercial Road, Melbourne, Victoria 3004, Australia; elsdon.storey@ med.monash.edu.au

Received 22 April 2004

Revised version received 8 March 2005

Accepted 25 March 2005

\section{REFERENCES}

1 Stevanin G, Bouslam N, Thobois S, et al. Spinocerebellar ataxia with sensory neuropathy (SCA25) maps to chromosome 2p. Ann Neurol 2004;55(1):97-104.

2 Chen DH, Brkanac Z, Verlinde CL, et al. Missense mutations in the regulatory domain of PKC gamma: a new mechanism for dominant nonepisodic cerebellar ataxia. Am J Hum Genet 2003;72(4):839-49.

3 Chen DH, Cimino PJ, Ranum L, et al. Prevalence of SCA14 and spectrum of PKC gamma mutations in a large panel of ataxia patients. Am J Hum Genet 2003;73(suppl):546

4 Brkanac $Z$, Bylenok L, Fernandez $M$, et al. A new dominant spinocerebellar ataxia linked to chromosome 19q13.4-qter. Arch Neurol 2002;59(8):1291-5.

5 Yamashita I, Sasaki H, Yabe I, et al. A novel locus for dominant cerebellar ataxia (SCA14) maps to a 10.2-cM interval flanked by D19S206 and D19S605 on chromosome 19q13.4-qter. Ann Neurol 2000;48(2):156-63.

6 Yabe I, Sasaki H, Chen DH, et al. Spinocerebellar ataxia type 14 caused by a mutation in protein kinase C gamma. Arch Neurol 2003;60(12):1749-51.

7 van de Warrenburg BP, Verbeek DS, Piersma SJ, et al. Identification of a novel SCA1 4 mutation in a Dutch autosomal dominant cerebellar ataxia family. Neurology 2003;61(12):1760-5.

8 Rivaud-Pechoux S, Durr A, Gaymard B, et al. Eye movement abnormalities correlate with genotype in autosomal dominant cerebellar ataxia type I. Ann Neurol 1998;43(3):297-302.

9 Buttner N, Geschwind D, Jen JC, et al. Oculomotor phenotypes in autosomal dominant ataxias. Arch Neurol 1998;55(10):1353-7.

10 Gomez CM, Thompson RM, Gammack JT, et al. Spinocerebellar ataxia type 6: gaze-evoked and vertical nystagmus, Purkinje cell degeneration, and variable age of onset. Ann Neurol 1997;42(6):933-50.

11 Knight MA, Gardner RJ, Bahlo M, et al. Dominantly inherited ataxia and dysphonia with dentate calcification: spinocerebellar ataxia type 20. Brain 2004; 127(Pt 5): 1172-81.

12 Day JW, Schut U, Moseley ML, et al. Spinocerebellar ataxia type 8: clinical features in a large family. Neurology 2000;55(5):649-57.

13 Worth PF, Giunti P, Gardner-Thorpe C, et al. Autosomal dominant cerebellar ataxia type III: linkage in a large British family to a $7.6-\mathrm{cM}$ region on chromosome 15q14-21.3. Am J Hum Genet 1999;65(2):420-6.

14 Miyoshi Y, Yamada T, Tanimura M, et al. A novel autosomal dominant spinocerebellar ataxia (SCA16) linked to chromosome 8q22.1-24.1. Neurology 2001;57(1):96-100.

15 Knight MA, Kennerson ML, Anney RJ, et al. Spinocerebellar ataxia type 15 (sca15) maps to 3p24. 2-3pter: exclusion of the ITPR1 gene, the human orthologue of an ataxic mouse mutant, Neurobiol Dis 2003;13(2):147-57.

16 Miller NR, Newman NJ, eds. Walsh and Hoyt's Clinical Neuroophthalmology, 5th ed. Baltimore: Lippincott Williams \& Wilkins, 1998

17 van Swieten JC, Brusse E, de Graaf BM, et al. A mutation in the fibroblast growth factor 14 gene is associated with autosomal dominant cerebellar ataxia [corrected]. Am J Hum Genet 2003;72(1):191-9.

18 Gundlfinger A, Kapthammer JP, Kruse F, et al. Different regulation of Purkinje cell dendritic development in cerebellar slice cultures by protein kinase Calpha and -beta. J Neurobiol 2003;57(1):95-109.

19 Verbeek DS, Knight MA, Harmison GG, et al. Protein kinase C gamma mutations in spinocerebellar ataxia 14 increase kinase activity and alter membrane targeting. Brain 2005; 128(Pt 2):436-42. 